\title{
Commutative or noncommutative spacetime? Two length scales of noncommutativity
}

\author{
R. Vilela Mendes* \\ IPFN, Instituto Superior Técnico, University of Lisboa, Av. Rovisco Pais 1, 1049-001 Lisboa, Portugal
}

(Received 9 January 2019; published 10 June 2019)

\begin{abstract}
The noncommutativity of the spacetime coordinates has been explored in several contexts, mostly associated with phenomena at the Planck length scale. However, approaching this question through deformation theory and the principle of stability of physical theories, one concludes that the scales of the noncommutativity of the coordinates and the noncommutativity of the generators of translations are independent. This suggests that the scale of the spacetime coordinates' noncommutativity could be larger than the Planck length. This paper attempts to explore the experimental perspectives to settle this question, either in the lab or by measurements of phenomena of cosmological origin.
\end{abstract}

DOI: 10.1103/PhysRevD.99.123006

\section{INTRODUCTION}

In this paper, I will address the following questions:

(1) Is spacetime a commutative or a noncommutative manifold?

(2) Can this question be decided in our time? That is, are there already sufficient experimental results and/or experimental instruments to decide?

To sharpen these questions, I will borrow from past results and from a few new ideas. The emphasis will be on the experimental perspectives.

To my knowledge, the first motivation to explore alternatives to the continuous commutative spacetime manifold was to cure the divergences arising in the perturbative treatment of quantum fields. In this context, several discrete time and/or discrete space models were proposed. However, these proposals violated Lorentz invariance, and it was Snyder [1] who made the first Lorentz-invariant proposal,

$$
\left[x_{\mu}, x_{\nu}\right]=\frac{i a^{2}}{\hbar} M_{\mu \nu}
$$

with $M_{\mu \nu}$ being the Lorentz group generators. However, the full Snyder algebra lacked translation invariance, and it was Yang [2] who pointed out that translation invariance would be recovered by interpreting the coordinate operators as

*rvilela.mendes@gmail.com; http://label2.ist.utl.pt/vilela/. Also at CMAFCIO, University of Lisboa, C6 - Faculdade de Ciências 1749-016 Lisboa, Portugal.

Published by the American Physical Society under the terms of the Creative Commons Attribution 4.0 International license. Further distribution of this work must maintain attribution to the author(s) and the published article's title, journal citation, and DOI. Funded by SCOAP ${ }^{3}$. generators of linear transformations in five-dimensional de Sitter space.

In recent years, the noncommutativity of the spacetime coordinates, in the sense

$$
\left[x_{\mu}, x_{\nu}\right]=i \theta_{\mu \nu},
$$

where $\theta_{\mu \nu}$ is either a $c$-number or an operator, has been explored in many contexts (see, for example, Refs. [3-6] and references therein). Noncommutative spacetime manifolds and noncommutative geometry techniques appear naturally in the context of string and $M$-theory, but so far, they lack a solid experimental or compelling physical motivation. An exception might be the work in Refs. [7,8]. There, it is argued that attempts to localize events with extreme precision cause gravitational collapse, so that spacetime below the Planck scale has no operational meaning, leading to spacetime uncertainty relations. However, this compelling reasoning would imply that the noncommutativity and the associated fundamental length would be of the order of Planck's length $\lambda_{P}=\left(\frac{G \hbar}{c^{3}}\right) \simeq 1.6 \times 10^{-33} \mathrm{~cm}$, far removed from current experimental reach. However, nothing forbids that the noncommutativity of spacetime might occur at a bigger scale. Nevertheless, most recent discussions of the noncommutativity of spacetime take place in the framework of quantum gravity, and therefore at the Planck length scale (see, for example, the review in Ref. [9] and references therein).

An alternative approach to the question of the noncommutativity of spacetime is based on deformation theory and the stability of physical theories.

\section{A. Noncommutative spacetime by deformation theory}

In the construction of models for the natural world, only those model properties that are robust have a chance to be 
observed. It is unlikely that properties that are too sensitive to small changes of the parameters will be well described in the model. If a fine-tuning of the parameters is needed to reproduce some natural phenomenon, then the model is basically unsound, and its other predictions are expected to be unreliable. For this reason a good methodological point of view consists in focusing on the robust properties of the models or, equivalently, to consider only models which are stable, in the sense that they do not change, in a qualitative manner, when some parameter changes. This is what has been called the stability of physical theories principle (SPTP) [10].

The stable-model point of view led in the field of nonlinear dynamics to the rigorous notion of structural stability [11,12]. As pointed out by Flato [13] and Faddeev [14], the same pattern seems to occur in the fundamental theories of nature. In particular, the passages from nonrelativistic to relativistic and from classical to quantum mechanics may be interpreted as transitions from two unstable theories to two stable ones. The stabilization of nonrelativistic mechanics corresponds to the deformation of the unstable Galileo algebra to the stable Lorentz algebra, and quantum mechanics arises as the stabilization of the Poisson algebra to the stable Moyal algebra [15]. However, when the generators of the Lorentz and the quantum mechanics Heisenberg algebra $\left\{M^{\mu \nu}, x^{\mu}, p^{\mu}\right\}$ are joined together, one finds out that the resulting PoincaréHeisenberg algebra is not stable either.

The Poincaré-Heisenberg algebra is deformed $[16,17]$ to the stable algebra $\Re_{\ell, \phi}=\left\{M^{\mu \nu}, p^{\mu}, x^{\mu}, \Im\right\}$ defined by the commutators

$$
\begin{aligned}
{\left[M^{\mu \nu}, M^{\rho \sigma}\right] } & =i\left(M^{\mu \sigma} \eta^{\nu \rho}+M^{\nu \rho} \eta^{\mu \sigma}-M^{\nu \sigma} \eta^{\mu \rho}-M^{\mu \rho} \eta^{\nu \sigma}\right) \\
{\left[M^{\mu \nu}, p^{\lambda}\right] } & =i\left(p^{\mu} \eta^{\nu \lambda}-p^{\nu} \eta^{\mu \lambda}\right) \\
{\left[M^{\mu \nu}, x^{\lambda}\right] } & =i\left(x^{\mu} \eta^{\nu \lambda}-x^{\nu} \eta^{\mu \lambda}\right) \\
{\left[p^{\mu}, x^{\nu}\right] } & =i \eta^{\mu \nu} \Im \\
{\left[x^{\mu}, x^{\nu}\right] } & =-i \epsilon \ell^{2} M^{\mu \nu} \\
{\left[p^{\mu}, p^{\nu}\right] } & =-i \epsilon^{\prime} \phi^{2} M^{\mu \nu} \\
{\left[x^{\mu}, \Im\right] } & =i \epsilon \ell^{2} p^{\mu} \\
{\left[p^{\mu}, \Im\right] } & =-i \epsilon^{\prime} \phi^{2} x^{\mu} \\
{\left[M^{\mu \nu}, \Im\right] } & =0
\end{aligned}
$$

which, according to the SPTP paradigm, one would expect to be a more accurate model. The stabilizing deformation introduces two new parameters $\ell^{2}, \phi^{2}$ and two signs $\epsilon, \epsilon^{\prime}$. The signs have physical relevance. For example, in the $\ell^{2} \neq 0$ case, if $\epsilon=+1$, time is discretely quantized, and if $\epsilon=-1$, it will be a space coordinate that has a discrete spectrum.

An important point that this deformation [16] of the Poincaré-Heisenberg algebra puts in evidence is the independence of the deformation parameters $\ell$ (associated with the noncommutativity of the spacetime coordinates) and $\phi$ (associated with the noncommutativity of momenta).

The stable algebra $\Re_{\ell, \phi}=\left\{x^{\mu}, M^{\mu \nu}, p^{\mu}, \Im\right\}$, to which the Poincaré-Heisenberg algebra has been deformed, is isomorphic to the algebra of the six-dimensional pseudoorthogonal group with metric

$$
\eta_{a a}=\left(1,-1,-1,-1, \epsilon, \epsilon^{\prime}\right), \quad \epsilon, \epsilon^{\prime}= \pm 1
$$

Both $\ell$ and $\phi^{-1}$ have dimensions of length. However, they might have different physical statuses and interpretations. Whereas $\ell$ might be considered as a fundamental length and a new constant of nature, $\phi^{-1}$, being associated with the noncommutativity of the generators of translation of the Poincare group, is associated with the local curvature of the spacetime manifold, ${ }^{1}$ and therefore is a dynamical quantity related to the local intensity of the gravitational field.

The two deformations - the one on the right-hand side of $\left[p^{\mu}, p^{\nu}\right]$ and the one on the right-hand side of $\left[x^{\mu}, x^{\nu}\right]$ 一are independent from each other. Being associated with the local gravitational field, it is natural that the scale of the deformation on the right-hand side of the $\left[p^{\mu}, p^{\nu}\right]$ commutator be the Planck length scale $\left(10^{-33} \mathrm{~cm}\right)$. However, there is no reason for the other one to have the same length scale. A basic conjecture that will be explored in this paper is that $\ell$ is much larger than $\phi^{-1}$. In particular, a deformed tangent space would correspond to take the limit $\phi^{-1} \rightarrow \infty$, obtaining

$$
\left.\left[p^{\mu}, p^{\nu}\right]\right|_{\phi^{-1} \rightarrow \infty} \rightarrow 0 \quad \text { and }\left.\quad\left[x^{\mu}, \Im\right]\right|_{\phi^{-1} \rightarrow \infty} \rightarrow 0
$$

with all the other commutators being the same as in Eq. (3), leading to the tangent space algebra ${ }^{2} \Re_{\ell, \infty}=$ $\left\{x^{\mu}, M^{\mu \nu}, \bar{p}^{\mu}, \bar{\Im}\right\}$.

The stable Poincaré-Heisenberg algebra in Eq. (3), obtained in Ref. [16], corresponds to a minimal deformation of the classical Poincaré-Heisenberg algebra. In Ref. [16], it is also pointed out that this deformation, while not being unique, is the one that seems to be the most reasonable from a physical point of view. Chryssomalakos and Okon's [18] (see also Refs. [19,20]) later careful analysis has then found the structure of the most general deformations of the Heisenberg-Poincaré algebra. This is summarized in the Appendix with a critical analysis of the physical reasoning behind the choice of the deformation in Eq. (3).

\footnotetext{
${ }^{1}$ In a de Sitter context, $\phi$ would be the inverse of the (local) curvature radius.

${ }^{2} \bar{p}^{\mu}, \bar{\Im}$ denotes the tangent space $\left(\phi^{-1} \rightarrow \infty\right)$ limits of the operators, not to be confused with the physical $p^{\mu}, \mathfrak{s}$ operators. According to the deformation-stability principle, they are stable physical operators only when $\phi^{-1}$ is finite - that is, when gravity is turned on.
} 
A first question of interest on the deformed algebras is the form of the dispersion relations. For the deformed tangent space algebra $\Re_{\ell, \infty}=\left\{x^{\mu}, M^{\mu \nu}, \bar{p}^{\mu}, \overline{\mathfrak{s}},\right\}$, it is

$$
\left(\bar{p}^{0}\right)^{2}-\left(\bar{p}^{1}\right)^{2}-\left(\bar{p}^{2}\right)^{2}-\left(\bar{p}^{3}\right)^{2}=\bar{Q}_{2},
$$

the same as in the Poincaré algebra, because this algebra is unchanged in $\Re_{\ell, \infty}, \bar{Q}_{2}=m^{2}$ being the quadratic Casimir operator.

For $\Re_{\ell, \phi}=\left\{x^{\mu}, M^{\mu \nu}, p^{\mu}, \Im\right\}$, it is

$$
P^{2}+\epsilon^{\prime} \phi^{2}\left(J^{2}-K^{2}\right)=Q_{2},
$$

with $P^{2}=p_{\mu} p^{\mu}, J^{i}=\frac{1}{2} \varepsilon^{i j k} M_{j k}, K^{i}=M^{i 0}$, and $Q_{2}$ being the quadratic Casimir operator for $\operatorname{SO}(3,2)\left(\epsilon^{\prime}=+1\right)$ or $S O(4,1)\left(\epsilon^{\prime}=-1\right)$.

The fact that the right-hand side of the commutator $\left[x^{\mu}, x^{\nu}\right]$ is a tensor operator rather than a $c$-number implies that most spacetime global symmetries are preserved (see, for example, Ref. [21]).

The deformed algebra [Eq. (3)] and its tangent space limit [Eq. (5)] have far-reaching consequences for the geometry of spacetime $[22,23]$, the dimension of the associated differentiable algebra, the interactions of connection-related quantum fields [24], and the Dirac equation [25]. Here, however, I will concentrate mostly on possible experimental tests and estimates of the value of the deformation parameters.

In the past, noncommutativity of the spacetime coordinates has been mostly associated with quantum gravity effects and the Planck length scale. Although, as pointed out in Ref. [9], some particular physical situations might greatly amplify the effects, the emphasis on the Plancklength-scale nature of the noncommutativity has precluded the search for laboratory-scale effects. The point of view proposed in this paper is that the formal independence of the deformation parameters $\ell$ and $\phi^{-1}$ suggests that these two length scales are naturally independent, and therefore it makes sense to look in the lab for the possibility of noncommutative effects at a scale larger than the Planck length.

\section{NONCOMMUTATIVE SPACETIME: EXPERIMENTAL PERSPECTIVES}

From the commutator $\left[x^{\mu}, x^{\nu}\right]=-i \epsilon \ell^{2} M^{\mu \nu}$ or from a more general one, $\left[x_{\mu}, x_{\nu}\right]=i \theta_{\mu \nu}$, one concludes that in the noncommutative case, the spacetime coordinates cannot be treated in isolation, and that at least an extra operator is involved in all calculations in the spacetime manifold. In the $\epsilon=+1$ case, the spacetime manifold is locally isomorphic to $S O(3,2)$, and in the $\epsilon=-1$ case, to $S O(4,1)$. Convenient tools for calculation are the representations of these algebras as operators on the corresponding cones (see Ref. [22] and the Appendixes in Refs. [23,26]), irreducible representations of these algebras playing the role of "points" in their noncommutative geometry.

Here, one analyzes a few situations where the noncommutativity of spacetime might be tested and measured, as well as some of the instances where such tests seem at present to be unfeasible. When the nature of the noncommutativity is left essentially unspecified, as in $\left[x_{\mu}, x_{\nu}\right]=$ $i \theta_{\mu \nu}$, it is difficult to obtain clearly testable predictions. Therefore, here, as a working principle, use will always be made of the commutation relations in Eq. (3) - in particular, in the tangent space limit [Eq. (5)].

\section{A. Measuring speed}

In the noncommutative context, space and time being noncommutative coordinates, they cannot be simultaneously diagonalized, and speed can only be defined in terms of expectation values - that is,

$$
v_{\psi}^{i}=\frac{1}{\left\langle\psi_{t}, \psi_{t}\right\rangle} \frac{d}{d t}\left\langle\psi_{t}, x^{i} \psi_{t}\right\rangle,
$$

where $\psi$ is a state with a small dispersion of momentum around a central value $p$. At time zero,

$$
\psi_{0}=\int\left|k^{0} \vec{k} \alpha\right\rangle f_{p}(k) d^{3} k
$$

where $k^{0}=\sqrt{|\vec{k}|^{2}+m^{2}}, \alpha$ stands for the quantum numbers associated with the little group of $k$, and $f_{p}(k)$ is a normalized function peaked at $k=p$.

In Ref. [27] a first-order (in $\ell^{2}$ ) derivation of the speed corrections was obtained. Here, a more complete treatment will be done. To obtain $\psi_{t}$, one applies to $\psi_{0}$ the time-shift operator, which is not $e^{-i a p^{0}}$, because

$$
e^{-i a p^{0}} t e^{i a p^{0}}=t+a \mathfrak{s}
$$

follows from

$$
\left[p^{0}, t\right]=i \mathfrak{\Im},
$$

whereas a time-shift generator $\Upsilon$ should satisfy

$$
[\Upsilon, t]=i \mathbf{1}
$$

Here, the calculations are carried out in the $\Re_{\ell, \infty}$ algebra. To implement the commutation relations of the deformed tangent space algebra $\Re_{\ell, \infty}=\left\{x^{\mu}, M^{\mu \nu}, \bar{p}^{\mu}, \bar{\Im}\right\}$, we use a basis where the five-variable set $\left(\bar{p}^{\mu}, \bar{\Im}\right)$ is diagonalized. ${ }^{3}$ In this basis, the commutation relations are realized by

\footnotetext{
${ }^{3}$ Notice that it is only in the tangent space algebra $\Re_{\ell, \infty}$ that the operators $\left(\bar{p}^{\mu}, \bar{\Im}\right)$ may be simultaneously diagonalized, not in the full algebra $\Re_{\ell, \phi}$.
} 


$$
\begin{aligned}
x^{\mu} & =i\left(\epsilon \ell^{2} \bar{p}^{\mu} \frac{\partial}{\partial \bar{\Im}_{s}}-\bar{\Im} \frac{\partial}{\partial \bar{p}_{\mu}}\right), \\
M_{\mu \nu} & =i\left(\bar{p}_{\mu} \frac{\partial}{\partial \bar{p}^{\nu}}-\bar{p}_{\nu} \frac{\partial}{\partial \bar{p}^{\mu}}\right) .
\end{aligned}
$$

Then, one obtains the following time-shift operator $\Upsilon$ in Eq. (10), to all $\ell^{2}$ orders:

$$
\Upsilon=\frac{\bar{p}^{0}}{\overline{\mathfrak{s}}} \sum_{k=0}(-\epsilon)^{k} \frac{\ell^{2 k}}{2 k+1}\left(\frac{\bar{p}^{0}}{\overline{\mathfrak{s}}}\right)^{2 k}
$$

To obtain this result, use may be made of $\left[t, \bar{\Im}^{-1}\right]=$ $-i \epsilon \ell^{2} \bar{p}^{0} \overline{\mathfrak{s}}^{-2}$, which follows from $\left[t, \overline{\mathfrak{s}}^{-1} \overline{\mathfrak{s}}^{-1}\right]=0$ and $[t, \bar{\Im}]=i \epsilon \ell^{2} \bar{p}^{0}$. Alternatively, one may check that Eq. (12) satisfies Eq. (10) using the representation (11) to obtain

$$
\begin{aligned}
{\left[\Upsilon, x^{0}\right]=} & i \sum_{k=0}\left\{(-\epsilon)^{k} \ell^{2 k}\left(\frac{\bar{p}^{0}}{\overline{\mathfrak{\Im}}}\right)^{2 k}\right. \\
& \left.-(-\epsilon)^{k+1} \ell^{2 k+2}\left(\frac{\bar{p}^{0}}{\overline{\mathfrak{s}}}\right)^{2 k+2}\right\} .
\end{aligned}
$$

More compact forms of the time-shift operator are

$$
\Upsilon=\left\{\begin{array}{ll}
\frac{1}{\ell} \tan ^{-1}\left(\ell \frac{\bar{p}^{0}}{\bar{\Im}}\right) & \epsilon=+1 \\
\frac{1}{\ell} \tanh ^{-1}\left(\ell \frac{\bar{p}^{0}}{\bar{\Im}}\right) & \epsilon=-1
\end{array} .\right.
$$

Now, one computes the time derivative of the expectation value of $x^{i}$ on the time-shifted state

$$
\psi_{t}=\int \exp (-i t \Upsilon)\left|\tilde{k}^{0} \tilde{k}^{i} \alpha\right\rangle f_{p}(\tilde{k}) d^{3} \tilde{k}
$$

From Eqs. (11) and (13), one has

$$
x^{i} e^{-i t \Upsilon}=e^{-i t \Upsilon} t \frac{\bar{p}^{i}}{\bar{p}^{0}} \frac{1-\epsilon \ell^{2}\left(\frac{\bar{p}^{0}}{\bar{\Im}}\right)^{2}}{1+\epsilon \ell^{2}\left(\frac{\bar{p}^{0}}{\bar{\Im}}\right)^{2}} .
$$

Therefore, the wave-packet velocity is

$$
v_{\psi}=\frac{\bar{p}}{\bar{p}^{0}} \frac{1-\epsilon \ell^{2}\left(\frac{\bar{p}^{0}}{\bar{\Im}}\right)^{2}}{1+\epsilon \ell^{2}\left(\frac{\bar{p}^{0}}{\bar{\Im}}\right)^{2}},
$$

a result that holds to all $\ell^{2}$ orders in $\Re_{\ell, \infty}$. In leading order, it is $v_{\psi} \simeq \frac{\bar{p}}{\bar{p}^{0}}\left(1-2 \epsilon \ell^{2}\left(\frac{\bar{p}^{0}}{\bar{J}}\right)^{2}\right)$. Notice that the correction is negative or positive, depending on the sign of $\epsilon$. For example, a massless-particle wave packet would be found to travel slower or faster than $c$ according to whether $\epsilon=$ +1 (quantized time) or $\epsilon=-1$ (quantized space). Also notice that this deviation from $c$, for the velocity of the massless-particle wave packet, implies no violation of relativity. Both the Lorentz and the Poincaré groups are still exact symmetries in $\Re_{\ell, \infty}$, and the velocity corrections do not arise from modifications of the dispersion relation for elementary states, which still is

$$
\left(\bar{p}^{0}\right)^{2}=(\overrightarrow{\bar{p}})^{2}+m^{2}
$$

but from the noncommutativity of time and space.

Now, some of the existing experimental results will be analyzed to find bounds on the value of $\ell$ (a fundamental time or a fundamental length).

In the corrected 2012 OPERA data [28] for $17 \mathrm{GeV}$ neutrinos, the reported result is

$$
\left|\frac{v-c}{c}\right|=\left(2.7 \pm 3.1 \text { (stat.) } \begin{array}{l}
+3.4 \\
-3.3
\end{array} \text { (sys.) }\right) \times 10^{-6}
$$

From

$$
\left|2 \epsilon \ell^{2}\left(\frac{\bar{p}^{0}}{\overline{\mathfrak{s}}}\right)^{2}\right| \leq 3 \times 10^{-6},
$$

with $\bar{p}^{0}=17 \mathrm{GeV}$ and the eigenvalue of the operator $\overline{\mathfrak{s}}$, on the right-hand side of the Heisenberg algebra, ${ }^{4}$ set to $\bar{\Im}=1$, it follows 5

$$
\ell \leq 1.4 \times 10^{-18} \mathrm{~cm}
$$

or, equivalently, for the elementary time,

$$
\tau \leq 0.5 \times 10^{-28} \mathrm{sec} .
$$

From the MINOS [30] data, with the neutrino spectrum peaked at $\bar{p}^{0}=3 \mathrm{GeV}$,

$$
\begin{gathered}
\left|\frac{v-c}{c}\right|=(5.1 \pm 2.9) \times 10^{-5}, \\
\left|2 \epsilon \ell^{2}\left(\frac{\bar{p}^{0}}{\bar{s}}\right)^{2}\right| \leq 5 \times 10^{-5},
\end{gathered}
$$

one obtains

$$
\ell \leq 3.3 \times 10^{-17} \mathrm{~cm}, \quad \tau \leq 10^{-27} \mathrm{sec} .
$$

Assuming a delay of at most a couple of hours between the neutrino and the visible-light outbursts from the SN1987A supernova, several authors [31-33] have estimated

\footnotetext{
${ }^{4}$ In the framework of the representations of some subalgebras [29] of Eq. (3), an explicit representation of $\bar{\Im}$ as $\bar{\Im}=\left(1+\ell^{2} \bar{p}^{2}\right)^{1 / 2}$ is possible. However, this does not change the $O\left(\ell^{2}\right)$ wave-packet speed correction.

${ }^{5}$ Notice that the correction due to a neutrino mass $\sim 2 \mathrm{eV}$ is smaller, of order $10^{-19}$.
} 


$$
\left|\frac{v-c}{c}\right|<2 \times 10^{-9}
$$

which with $\bar{p}^{0} \approx 10 \mathrm{MeV}$ would lead to

$$
\ell<6 \times 10^{-17} \mathrm{~cm}, \quad \tau<2 \times 10^{-27} \mathrm{sec} .
$$

One sees that all this data is compatible with a value $\ell \lesssim 10^{-18} \mathrm{~cm}$ or $\tau \lesssim 0.3 \times 10^{-28} \mathrm{sec}$. Using this value, one also sees that the effect is extremely small for visible light. For example, with $\bar{p}^{0}=3 \mathrm{eV}$ and $\ell=10^{-18} \mathrm{~cm}$, one obtains

$$
\left|\frac{v-c}{c}\right|<4.6 \times 10^{-26}
$$

These are results for elementary states. For slow macroscopic matter, instead of Eq. (7), the state is

$\psi_{0}(P)=\int\left|k_{1}, k_{2}, \ldots, k_{N}\right\rangle f_{P}\left(k_{1}, k_{2}, \ldots, k_{N}\right) d k_{1} d k_{2} \ldots d k_{N}$.

Whenever the coupling energy of the elementary constituents of the macroscopic body is much smaller than their rest masses, one may factorize the time-shift operator:

$e^{-i \uparrow \Upsilon}\left|k_{1}, k_{2}, \ldots, k_{N}\right\rangle=\left|e^{-i t \Upsilon_{1}} k_{1}, e^{-i t \Upsilon_{2}} k_{2}, \ldots, e^{-i t \Upsilon_{N}} k_{N}\right\rangle$.

Therefore, for a nonrelativistic body $\bar{p}^{0} \simeq m_{p}$ (the proton mass $m_{p}=938 \mathrm{MeV}$ ) leads, with $\ell=10^{-18} \mathrm{~cm}$, to a speed correction

$$
\left|\frac{v-\frac{\bar{p}}{\bar{p}^{0}}}{\frac{\bar{p}}{\bar{p}^{0}}}\right|=0.452 \times 10^{-8} .
$$

It does not sound like much; however, for a nominal velocity $\frac{\bar{p}}{\bar{p}^{0}}=10 \mathrm{~km} / \mathrm{sec}$, it would lead after one year to a deviation of $1.4 \mathrm{~km}$.

All the above bounds are much larger than the Planck timescale, and improving them seems in reach of present experimental techniques. In particular, it would be interesting to refine the neutrino wave-packet speed measurements, preferably with a larger baseline.

Presumably, the best way to test the speed corrections arising from noncommutativity would be to consider phenomena involving cosmological distances. This is also the point of view of many authors when looking for light velocity modifications as a probe of Lorentz-invariance violation (LIV) (see Refs. [34-36] and references therein). In particular, special attention has been devoted to gammaray bursts (GRB). Notice, however, that in the present paper no LIV is implied; it is the noncommutativity that impacts the group velocity of massless-particle wave packets. In any case, the LIV estimates of these authors may in some cases be carried over to the noncommutativity framework, and I will comment on that later.

As will be seen, the calculation of cosmological distances (angular diameter and luminosity distance) is affected by the energy-dependent wave-packet speed corrections.

One uses the Robertson-Walker metric

$(d s)^{2}=(d t)^{2}-a^{2}(t)\left\{\frac{(d r)^{2}}{1-K r^{2}}+r^{2}\left((d \theta)^{2}+\sin ^{2} \theta(d \phi)^{2}\right)\right\}$

$(c=\hbar=1)$.

For a massless wave packet with central energy $E$ moving radially at speed $v(E)$,

$$
v(E(t)) \frac{d t}{a(t)}=\frac{d r}{\sqrt{1-K r^{2}}},
$$

with, in leading $\ell^{2}$ order

$$
v(E(t))=1-2 \epsilon \ell^{2} E^{2}(t)=1-8 \pi^{2} \epsilon \ell^{2} \frac{1}{\lambda^{2}(t)},
$$

$\lambda$ being the wavelength. Considering now two crests in the central frequency of the packet, using Eq. (30),

$$
\frac{v\left(E_{0}\right)}{a\left(t_{0}\right)} \lambda_{0}=\frac{v\left(E_{e}\right)}{a\left(t_{e}\right)} \lambda_{e},
$$

which in leading $\ell^{2}$ order is

$$
\frac{\lambda_{e}}{\lambda_{0}}\left\{1-8 \pi^{2} \epsilon \ell^{2}\left(\frac{1}{\lambda_{e}^{2}}-\frac{1}{\lambda_{0}^{2}}\right)\right\}=\frac{a\left(t_{e}\right)}{a\left(t_{0}\right)},
$$

$\lambda_{e}$ being the emitted wavelength at time $t_{e}$ and $\lambda_{0}$ the received one at time $t_{0}$. Defining $1+z=\frac{\lambda_{0}}{\lambda_{e}}$,

$$
\frac{a\left(t_{0}\right)}{a\left(t_{e}\right)}=\frac{1+z}{\Gamma\left(\lambda_{0}, z\right)},
$$

with

$$
\Gamma\left(\lambda_{0}, z\right)=1-8 \pi^{2} \epsilon \frac{\ell^{2}}{\lambda_{0}^{2}} z(z+2) .
$$

Therefore, the relation between the ratio $\frac{a\left(t_{0}\right)}{a\left(t_{e}\right)}$ and the redshift $z$ depends on the frequency that is being observed-that is, when using integration over redshift, to obtain the propagation time, one should take into account the wavelength for which the redshift is being measured. From Eq. (33), one obtains 


$$
d t=\frac{1}{H(t)}\left(\frac{d \log \Gamma\left(\lambda_{0}, z\right)}{d z}-\frac{1}{1+z}\right) d z
$$

with $H(t)$ being the Hubble parameter,

$$
H(t)=\frac{\dot{a}(t)}{a(t)} .
$$

The Friedmann equation becomes

$$
\begin{aligned}
\frac{H(t)}{H_{0}} & =\sqrt{\sum_{i} \Omega_{i, 0}\left(\frac{1+z}{\Gamma\left(\lambda_{0}, z\right)}\right)^{3}+\Omega_{\mathrm{rad}, 0}\left(\frac{1+z}{\Gamma\left(\lambda_{0}, z\right)}\right)^{4}+\Omega_{K, 0}\left(\frac{1+z}{\Gamma\left(\lambda_{0}, z\right)}\right)^{2}+\Omega_{\Lambda, 0}} \\
& =\sqrt{E\left(\lambda_{0}, z\right)},
\end{aligned}
$$

with the $\Omega$ constants related, respectively, to matter, radiation, curvature, and vacuum energy. The dependence on $\lambda_{0}$ means that the redshift $z$ is computed from the received light at wavelength $\lambda_{0}$.

The dependence on $\lambda_{0}$ would also have an impact on estimates of the age of the Universe:

$$
t_{0}=\frac{1}{H_{0}} \int_{0}^{\infty} \frac{d z}{\sqrt{E\left(\lambda_{0}, z\right)}}\left(\frac{1}{1+z}-\frac{d \log \Gamma\left(\lambda_{0}, z\right)}{d z}\right) .
$$

For the angular diameter $d_{A}$ and luminosity $d_{L}$ distances, one has

$$
\begin{gathered}
d_{A}=\frac{\Gamma\left(\lambda_{0}, z\right)}{1+z} F_{K}\left(\frac{1}{H_{0}} \int_{0}^{z} \frac{(1+z)\left(\frac{1}{1+z}-\frac{d \log \Gamma\left(\lambda_{0}, z\right)}{d z}\right)\left(1-8 \pi^{2} \epsilon \frac{\ell^{2}(1+z)^{2}}{\lambda_{0}^{2}}\right)}{\Gamma\left(\lambda_{0}, z\right) \sqrt{E\left(\lambda_{0}, z\right)}} d z\right), \\
d_{L}=\frac{(1+z)^{2}}{\Gamma^{2}\left(\lambda_{0}, z\right)} d_{A},
\end{gathered}
$$

with $F_{K}=\left\{\begin{array}{c}\sin \\ 1 \\ \text { sinh }\end{array}\right.$ for $K=\left\{\begin{array}{c}1 \\ 0 . \\ -1\end{array}\right.$.

With these results, some experimental information might be obtained from cosmological data. As an example, consider the spectral lags [37-41] in gamma-ray bursts (GRB). The spectral lag is defined as the difference in the time of arrival of high- and low-energy photons. It is considered positive when the high-energy photons arrive earlier than the low-energy ones. The spectral lags being associated with the spectral evolution during the prompt GBR phase, one expects different source types to have different intrinsic lags at the source. In addition, due to the complex nature of the gamma-ray peak structure, the spectral lags, obtained from delayed correlation measurements, have large error bars. Nevertheless, they allow access to timescales not achievable in the labs, and it might be worthwhile to test whether the lags are also affected by energy-dependent propagation effects. A few simple hypotheses will be made about the relation between the lag in the production of gamma rays at the source and their observation at Earth. Let us consider two gamma pulses at different energies $E_{1}$ and $E_{2}\left(E_{2}>E_{1}\right)$ produced with an intrinsic lag $\alpha^{(a)}$ at the source $a$.

If $T_{a}^{(1)}$ and $T_{a}^{(2)}$ are their propagation times from the source $a$ to Earth, the spectral lag would be

$$
\Delta t_{a}=T_{a}^{(1)}-T_{a}^{(2)}+\alpha^{(a)}
$$

From Eqs. (35) and (37),

$$
\begin{aligned}
T^{(i)}\left(\lambda_{0}^{(i)}, z\right)= & \frac{1}{H_{0}} \int_{0}^{z} d z^{\prime} \frac{1}{\sqrt{E\left(z^{\prime}\right)}} \\
& \times\left\{\frac{1}{1+z^{\prime}}-\frac{d}{d z^{\prime}} \log \Gamma\left(\lambda_{0}^{(i)}, z\right)\right\},
\end{aligned}
$$

with $\lambda_{0}^{(i)}$ being the wavelengths as observed at Earth and $E(z)$ defined in Eq. (37). Adopting the nowadays consensus cosmology $\Omega_{m, 0}=0.3, \Omega_{\Lambda, 0}=0.7, \Omega_{k, 0}=\Omega_{\mathrm{rad}, 0}=0$, $K=0, T^{(i)}\left(\lambda_{0}, z\right)$ becomes in leading $\frac{\ell^{2}}{\lambda_{0}^{2}}$ order

$$
T^{(i)}\left(\lambda_{0}^{(i)}, z\right) \simeq \frac{I_{1}(z)}{H_{0}}+\frac{4 \pi^{2} \epsilon \ell^{2}}{H_{0} \lambda_{0}^{(i) 2}} I_{2}(z),
$$

with the integrals $I_{1}$ and $I_{2}$ being

$$
\begin{aligned}
I_{1}(z)= & \int_{0}^{z} \frac{d z^{\prime}}{1+z^{\prime}} \frac{1}{\left(\Omega_{m, 0}\left(1+z^{\prime}\right)^{3}+\Omega_{\Lambda, 0}\right)^{\frac{1}{2}}}, \\
I_{2}(z)= & \int_{0}^{z} d z^{\prime} \\
& \times \frac{\Omega_{m, 0}\left(1+z^{\prime}\right)^{2}\left(z^{\prime 2}+2 z^{\prime}+4\right)+4\left(1+z^{\prime}\right) \Omega_{\Lambda, 0}}{\left(\Omega_{m, 0}\left(1+z^{\prime}\right)^{3}+\Omega_{\Lambda, 0}\right)^{\frac{3}{2}}} .
\end{aligned}
$$


TABLE I. Burst codes for the 24 gamma-ray bursts labeled by number in the plots of Figs. 1 and 2.

\begin{tabular}{llll}
\hline \hline 1 & GRB050401 & 13 & GRB080413B \\
2 & GRB050922C & 14 & GRB080605 \\
3 & GRB051111 & 15 & GRB080916A \\
4 & GRB060210 & 16 & GRB081222 \\
5 & GRB061007 & 17 & GRB090618 \\
6 & GRB061121 & 18 & GRB090715B \\
7 & GRB071010B & 19 & GRB090926B \\
8 & GRB071020 & 20 & GRB091024 \\
9 & GRB080319B & 21 & GRB091208B \\
10 & GRB080319C & 22 & GRB100621A \\
11 & GRB080411 & 23 & GRB100814A \\
12 & GRB080413A & 24 & GRB100906A \\
\hline \hline
\end{tabular}

From Eqs. (41) and (43), one sees that the lags are linear on $I_{2}(z)$,

$$
\Delta t_{a}=\frac{4 \pi^{2} \epsilon \ell^{2}}{H_{0}} I_{2}(z)\left(\frac{1}{\lambda_{0}^{(1) 2}}-\frac{1}{\lambda_{0}^{(2) 2}}\right)+\alpha_{a}^{(0)},
$$

for wavelengths at Earth or on $\frac{I_{2}(z)}{(1+z)^{2}}$ for energies at the source,

$$
\begin{aligned}
\Delta t_{a} & =\frac{4 \pi^{2} \epsilon \ell^{2}}{H_{0}} \frac{I_{2}(z)}{(1+z)^{2}}\left(\frac{1}{\lambda_{e}^{(1) 2}}-\frac{1}{\lambda_{e}^{(2) 2}}\right)+\alpha_{a}^{(0)} \\
& =\frac{\epsilon \ell^{2}}{H_{0}} \frac{I_{2}(z)}{(1+z)^{2}}\left(E_{e}^{(1) 2}-E_{e}^{(2) 2}\right)+\alpha_{a}^{(0)} .
\end{aligned}
$$

Thus, for fixed $\left(E_{e}^{(1) 2}-E_{e}^{(2) 2}\right)$, one may expect the data to be fitted by a few parallel lines, each one corresponding to a particular type of lag mechanism at the source. This analysis is similar to what has been done by other authors (see, for example, Refs. [35,42]) in the context of searches for LIV.

Let $H_{0}=70 \mathrm{~km} \mathrm{~s}^{-1}, \Omega_{m, 0}=0.3, \Omega_{\Lambda, 0}=0.7$, and to test the hypothesis, use the Swift BAT data in Ref. [40] for spectral lags of the source-frame bands $100-150 \mathrm{keV}$ and 200-250 kev $\left(E_{0}^{(1)}=\frac{125}{1+z}, E_{0}^{(2)}=\frac{225}{1+z}\right)$, selecting the 24 bursts for which the lags were computed with significance $1 \sigma$ or greater. Table I lists the correspondence of the numbers in the plot with the burst code.

Comparison of the data with Eq. (45) is performed by minimizing in $\beta$ and $\vec{\alpha}$ the function

$$
f(\beta, \vec{\alpha})=\sum_{i} \min _{\vec{\alpha}}\left\{y_{i}-\left(\beta x_{i}+\vec{\alpha}\right)\right\}^{2}
$$

for several dimensions of the vector $\vec{\alpha}$ (the vector of intrinsic lags). Here, the variables $y_{i}$ and $x_{i}$ are, respectively, the observed lags $\Delta t_{a}$ and $\frac{I_{2}(z)}{(1+z)^{2}}$. As the dimension of $\vec{\alpha}$ (the number of different lag types at the sources) increases, the fitting error, defined as

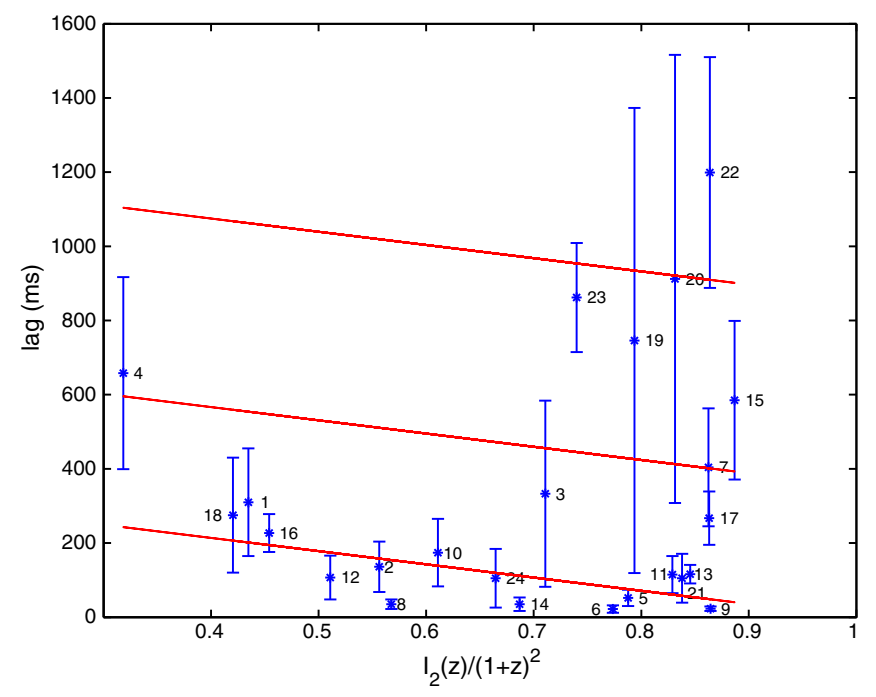

FIG. 1. Lags vs $\frac{I_{2}(z)}{(1+z)^{2}}$ for 24 GRBs with $1 \sigma$ significance or greater (data from Ref. [40]).

$$
e r=\frac{f(\beta, \vec{\alpha})}{\sum_{i} y_{i}^{2}}
$$

decreases. The fitting accuracy improves appreciably until the dimension of $\vec{\alpha}^{(0)}$ is equal to 3 , but not much afterwards.

Figure 1 shows the data points and the fitting lines for $\operatorname{dim} \vec{\alpha}^{(0)}=3$. The error is er $=0.05$. The slope $\beta$ is $\simeq-360$, corresponding with $\left(E_{1}^{2}-E_{2}^{2}\right)=35000 \mathrm{keV}^{2}$ to $\ell \simeq 0.95 \times 10^{-19} \mathrm{~cm}$ and $\epsilon=+1 \quad\left(\tau=3 \times 10^{-30} \mathrm{sec}\right)$. Notice that $I_{2}(z)$ grows with $z$, but not $\frac{I_{2}(z)}{(1+z)^{2}}$. As shown in Fig. 2, the result is quite similar when one restricts the data to the GBRs with significance $2 \sigma$ or greater.

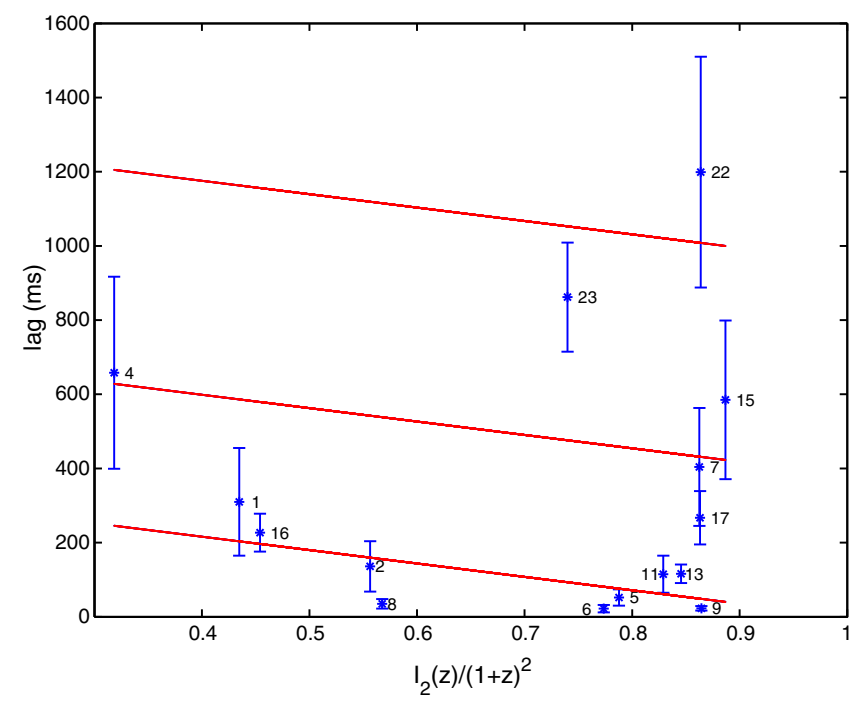

FIG. 2. Lags vs $\frac{I_{2}(z)}{(1+z)^{2}}$ for 15 GRBs with significance $2 \sigma$ or greater (data from Ref. [40]). 


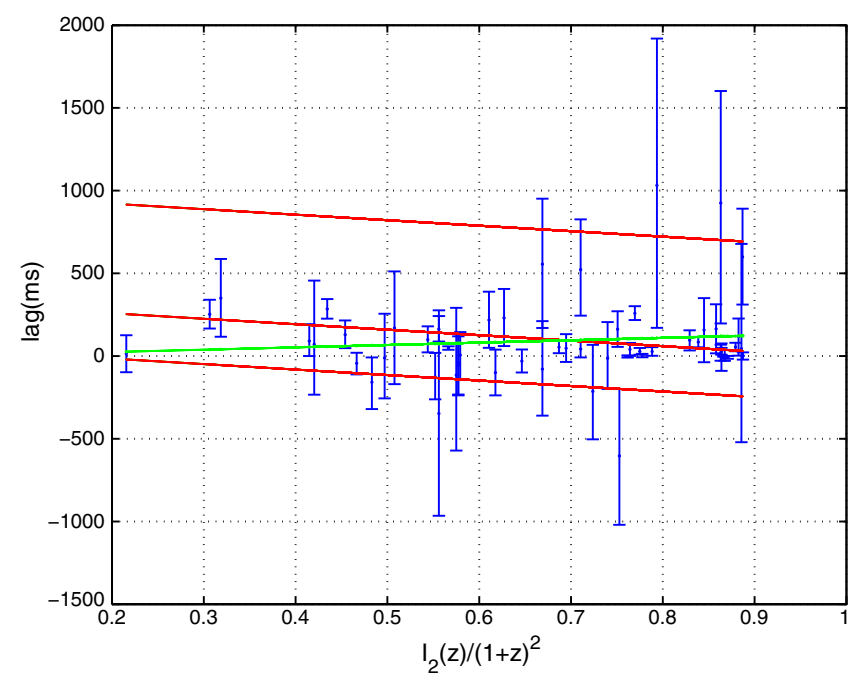

FIG. 3. Lags vs $\frac{I_{2}(z)}{(1+z)^{2}}$ for the 57 GRBs in Ref. [43] and fitting lines with three intersects (red) or one intersect (green).

Notice that $\epsilon=+1$ corresponds to higher-energy pulses traveling slower than lower-energy ones.

A larger set of GRB data with known redshifts is studied in Ref. [43]. The main difference from the analysis in Ref. [40] is the use of an asymmetric Gaussian model for the cross-correlation function to compute the spectral lags. Otherwise, the source frame energy bands (100-150 and 200-250 keV) are the same as in Ref. [40]. The same fitting technique as before was here applied to the 57 GRBs in Ref. [43] with the result shown in Fig. 3. For three intersects (dimension of $\vec{\alpha}^{(0)}=3$ ), the slope that is obtained is $\beta \simeq-330 \quad(e r=0.18)$, corresponding to $\ell \simeq 0.9 \times$ $10^{-19} \mathrm{~cm}, \epsilon=+1$, a result consistent with the one obtained before. Notice, however, that if instead of the dimension of $\vec{\alpha}^{(0)}=3$, one assumes a dimension of $\vec{\alpha}^{(0)}=1$, one obtains a worse fit ( $e r=0.87)$ and a quite different result-that is, $\ell \simeq 0$, the dash-dotted green line in Fig. 3. This is essentially what has been done in Ref. [44], with these authors concluding that there is no evidence for LIV. However, that hypothesis (dimension of $\vec{\alpha}^{(0)}=1$ ) assumes that all the intrinsic lags at the source are the same.

In Eq. (45), the $\alpha_{a}^{(0)}$ line intersects represent several classes of intrinsic lags as seen at Earth. It might be better to use the intrinsic lags $\alpha_{a}^{(e)}$ at the source-that is, replace $\alpha_{a}^{(0)}$ with $\alpha_{a}^{(0)}=\alpha_{a}^{(e)}(1+z)$. Then, one has

$$
\frac{\Delta t_{a}}{1+z}=\frac{\epsilon \ell^{2}}{H_{0}} \frac{I_{2}(z)}{(1+z)^{3}}\left(E_{e}^{(1) 2}-E_{e}^{(2) 2}\right)+\alpha_{a}^{(e)} .
$$

With this equation and the data in Ref. [43], one obtains the results shown in Fig. 4.

For the dimension of $\vec{\alpha}^{(e)}=3$, the slope is $\beta=-448$ $(e r=0.2)$, corresponding with $\left(E_{e}^{(1) 2}-E_{e}^{(2) 2}\right)=3.5 \times$ $10^{4} \mathrm{keV}^{2}$ to $\ell \simeq 1.06 \times 10^{-19} \mathrm{~cm}$. And, as before, a very

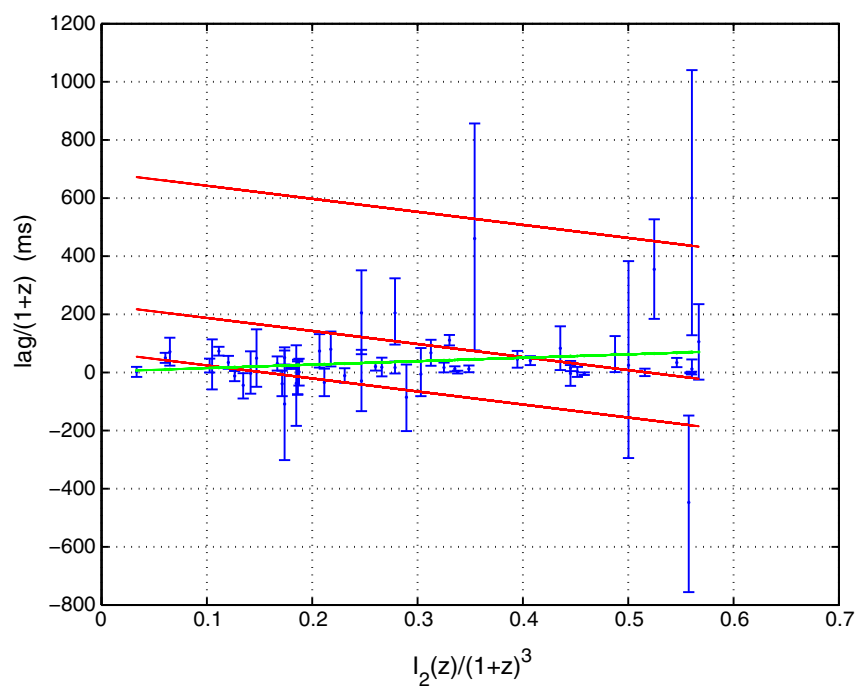

FIG. 4. Lags $/(1+z)$ vs $\frac{I_{2}(z)}{(1+z)^{3}}$ for the 57 GRBs in Ref. [43] and fitting lines with three intersects (red) or one intersect (green).

different result is obtained for a dimension of $\vec{\alpha}^{(e)}=1$ $(e r=0.9)$, the dash-dotted line in Fig. 4.

For short GRB pulses, intrinsic lags are in general considered smaller than those of long GRB pulses. Looking for eventual Lorentz-invariance violation (LIV), the authors in Ref. [45] have analyzed 15 short pulses (on the energy bands 50-100 and 150-200 keV), concluding that there is no evidence ${ }^{6}$ for energy dependence of the light propagation speed. Here, the same analyzing technique as described above has been applied to the same data with a different conclusion, as shown in Fig. 5.

The figure shows the fitting of the data assuming either a dimension of $\vec{\alpha}^{(e)}=3$ (red continuous lines) or a dimension of $\vec{\alpha}^{(e)}=1$ (dash-dotted green line). The slope is $\beta \simeq-230$, corresponding with $\left(E_{e}^{(1) 2}-E_{e}^{(2) 2}\right)=2.5 \times$ $10^{4} \mathrm{keV}^{2}$ to $\ell \simeq 0.9 \times 10^{-19} \mathrm{~cm}$. Notice that in this case, the slope obtained with one or three intersects is essentially the same, suggesting that for this set of pulses, the intrinsic lags are identical. The differences from the conclusions of the authors in Ref. [45] are not, of course, due to any mistake of these authors, but to the fact that they plot the data with respect to a $K(z)$ function, whereas here, according to the calculations above, the $z$ dependence is coded by the $I_{2}(z)$ function [Eq. (44)].

Of course, all these results, as well as the searches for LIV (see, for example, Ref. [36] and references therein), can only be taken as indicative or as establishing an upper bound on $\tau$ because of the large uncertainties on the calculation of the spectral lags, on the statistics of the GRB pulses, and even more, on the intrinsic spectral lags $\alpha_{a}$. However, if correct, they have some implications

\footnotetext{
${ }^{6}$ Actually, the authors' conclusion is that the quantum gravity scale $E_{Q G} \gtrsim 1.5 \times 10^{16} \mathrm{GeV}$, which would correspond to a scale $\ell \lesssim 1.3 \times 10^{-30} \mathrm{~cm}$.
} 


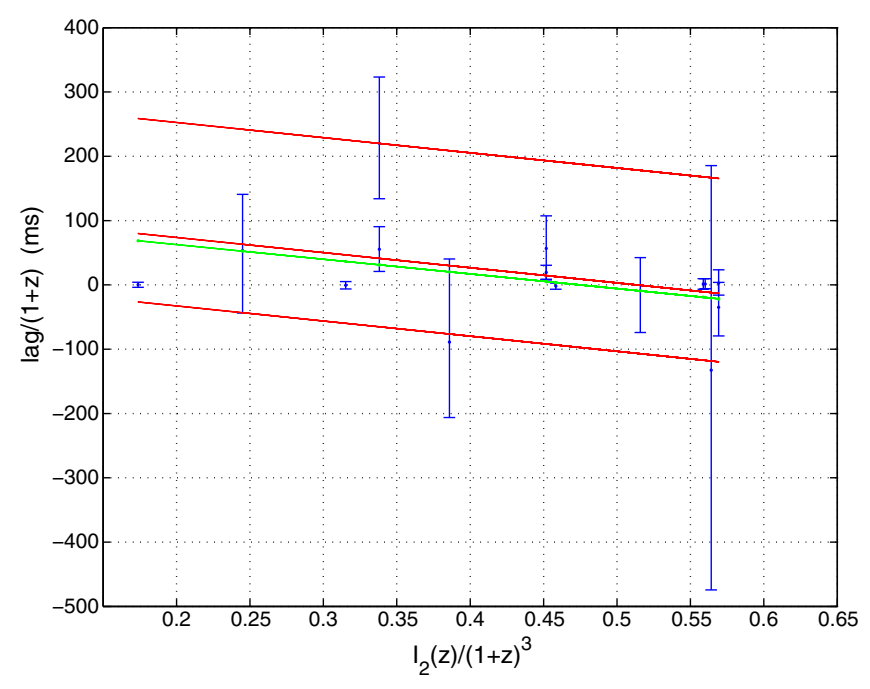

FIG. 5. Lags $/(1+z)$ vs $\frac{I_{2}(z)}{(1+z)^{3}}$ for the 15 short GRBs in Ref. [45] and fitting lines with three intersects (red) or one intersect (green).

concerning the observation of neutrino emissions from the GRB sources and also on the SN1987A observations. From the SN1987A supernova, neutrinos were observed in the range from 7.5 to $40 \mathrm{Mev}[31,32]$. Using Eq. (15) to obtain the propagation-time difference over 168000 light years, between visible light and neutrino packets of 10 and 40 Mev, with $\ell=10^{-19} \mathrm{~cm}$, one obtains $1.1 \times 10^{-3}$ and $1.8 \times 10^{-2} \mathrm{sec}$, respectively. Clearly, this does not change the estimate in Eq. (24). However, for GRBs at cosmological distances, the situation is different. From Eq. (35), $\ell=10^{-19} \mathrm{~cm}$, and $\epsilon=+1$, neutrinos of energy $40 \mathrm{Mev}$ would take 27 hours more than visible light to reach Earth from a source at $z=2$ redshift and 1.7 more hours from a source at redshift $z=1$. For $10 \mathrm{MeV}$ neutrinos, the results would be 1.7 hours and 1 hour, respectively. For $\ell=10^{-18} \mathrm{~cm}$, these numbers would be multiplied by 100 and also grow quadratically with the energy.

Recently, a very high-energy neutrino was observed from the direction of active galactic nuclei at cosmological distance $[46,47]$. If $\ell$ is in the range discussed above, the conclusion is that it could only have originated from a much earlier event, not a recent flare of gamma activity. Alternatively, if by some means its origin is proved to be coincident with recently observed gamma flares, that would mean that $\ell$ is much smaller than suggested here (that is, $\ell \leqslant 10^{-24} \mathrm{~cm}$ ). Notice, however, that dedicated searches [48-50] for neutrinos in close coincidence with GRB bursts found no or scarce evidence for them.

Wei et al. [51,52] analyzed a burst GRB160625B with unusually high photon statistics and a steep decline from positive lags to smaller ones with increasing photon energy in the range 8-20 MeV. They have fitted the spectral lag data using a power law for the intrinsic lag and a linear or quadratic term corresponding to the LIV correction. Here, the same data have been analyzed using also a power law

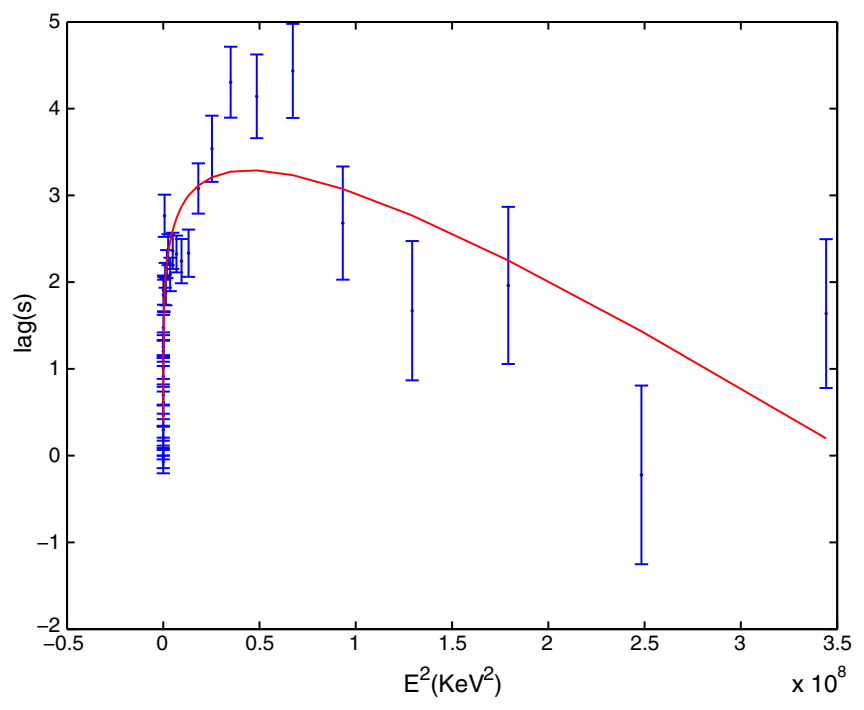

FIG. 6. Fitting the GRB160625B data to Eq. (47).

for the intrinsic lag together with the noncommutativity correction, namely

$$
l a g=\alpha E^{\beta}-\frac{\epsilon \ell^{2}}{H_{0}} I_{2}(1.41) E^{2} .
$$

The least-squares result is shown in Fig. 6. One sees that the fitting accuracy is rather poor, which is even more apparent using a linear $E^{2}$ axis than in the log-log plot used in Ref. [51]. Actually, the small statistical significance of the fitting using an equation of the type of Eq. (47) had already been pointed out in Ref. [53].

In fact, given the probable multiple shock mechanism of the GRB's generation, it is not likely for a continuous power dependence of the intrinsic lag to be a good hypothesis. It seems better to concentrate on the highenergy tail of the data and try the equation

$$
l a g=\alpha E^{0.18+\beta E^{2}}-\frac{\epsilon \ell^{2}}{H_{0}} I_{2}(1.41) E^{2} .
$$

This is used to fit the data between 5 and $20 \mathrm{GeV}$, the result being shown in Fig. 7.

The minimizing parameters are $\alpha=0.29, \quad \beta=$ $1.538 \times 10^{-10}$, and

$$
\frac{\epsilon \ell^{2}}{H_{0}} I_{2}(1.41)=7.794 \times 10^{-8},
$$

with 0.18 in the exponent being the value obtained in the fitting to Eq. (47). With $I_{2}(1.41)=4.3445$, one obtains from Eq. (49) $\ell=1.79 \times 10^{-21} \mathrm{~cm}$ or $\tau=0.597 \times 10^{-31} \mathrm{sec}$. This is 2 orders of magnitude smaller than obtained before, but there is the small significance of a result obtained with a single burst, compounded with the small quantitative 


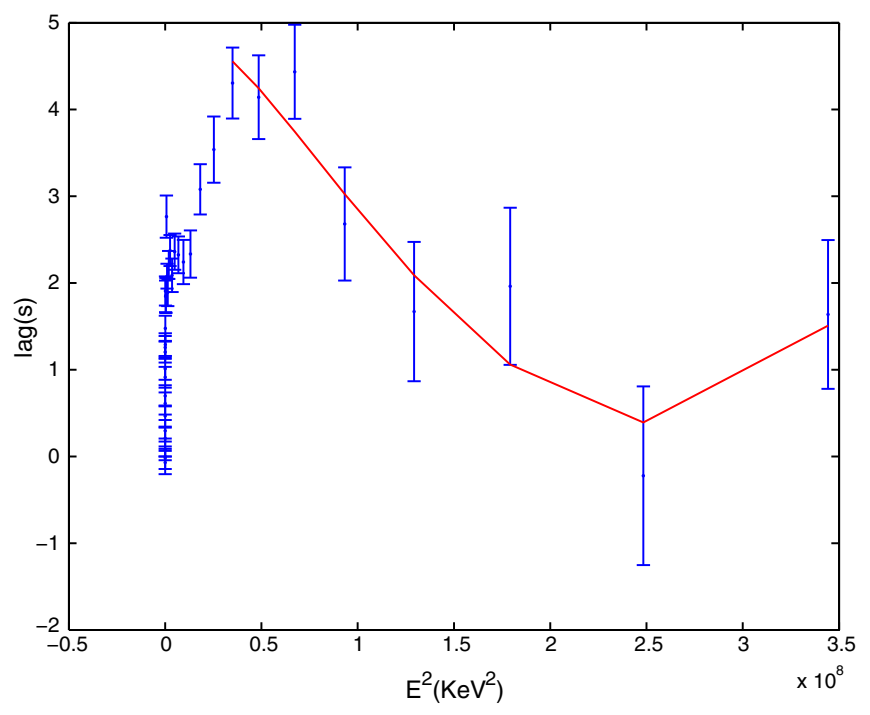

FIG. 7. Fitting the GRB160625B data between 5 and $20 \mathrm{GeV}$ by Eq. (48).

knowledge that still exists about the intrinsic lags at the source.

Finally, from Eq. (40), one may also estimate the impact of an energy dependent propagation speed on the calculation of the Hubble constant from observations at cosmological distances. Given the luminosity $L$ and the observed flux $F_{o}$ from a standard candle, the luminosity distance $d_{L}$ is

$$
d_{L}^{2}=\frac{L}{4 \pi F_{o}} .
$$

On the other hand, from Eqs. (39) and (40),

$$
d_{L}=\frac{1+z}{\Gamma\left(\lambda_{0}, z\right)} I(z)
$$

with

$I(z)=\frac{1}{H_{0}} \int_{0}^{z} \frac{(1+z)\left(\frac{1}{1+z}-\frac{d \log \Gamma\left(\lambda_{0}, z\right)}{d z}\right)\left(1-8 \pi^{2} \frac{\ell^{2}}{\lambda_{0}^{2}} \frac{(1+z)^{2}}{\Gamma^{2}\left(\lambda_{0}, z\right)}\right)}{\Gamma\left(\lambda_{0}, z\right) \sqrt{E\left(\lambda_{0}, z\right)}} d z$.

Therefore, given $d_{L}$ from Eq. (50), $H_{0}$ is obtained from

$$
H_{0}=\frac{(1+z) I_{0}(z)}{d_{L}}+\frac{\pi^{2} \epsilon \ell^{2}}{\lambda_{0}^{2}}(1+z)\left\{I_{3}(z)+8 z\left(z+2 I_{0}(z)\right)\right\}
$$

with

$$
\begin{aligned}
& I_{0}(z)=\int_{0}^{z} \frac{d z^{\prime}}{\left(\Omega_{m, 0}\left(1+z^{\prime}\right)^{3}+\Omega_{\Lambda, 0}\right)^{\frac{1}{2}}}, \\
& I_{3}(z)=\int_{0}^{z}\left\{\frac{2 z^{\prime 2}+8 z^{\prime}+3}{\left(\Omega_{m, 0}\left(1+z^{\prime}\right)^{3}+\Omega_{\Lambda, 0}\right)^{\frac{1}{2}}}-\frac{\left.12 z^{\prime}\left(z^{\prime}+2\right) \Omega_{m, 0}\right)\left(1+z^{\prime}\right)^{3}}{\left(\Omega_{m, 0}\left(1+z^{\prime}\right)^{3}+\Omega_{\Lambda, 0}\right)^{\frac{3}{2}}}\right\} d z^{\prime}
\end{aligned}
$$

Then, the correction to the $H_{0}$ calculation is

$$
\frac{H_{0}(\ell \neq 0)}{H_{0}(\ell=0)}=1+\frac{\pi \epsilon \ell^{2}}{\lambda_{0}^{2}}\left\{\frac{I_{3}(z)}{I_{0}(z)}+8 z(z+2)\right\} .
$$

However, for example, for $z=0.5$, this would be $1+$ $17.3 \frac{\pi \epsilon \ell^{2}}{\lambda_{0}^{2}}$, which for visible light $\left(\lambda_{0}=3.9-7 \times 10^{-9} \mathrm{~cm}\right)$ would be too small to be of any importance. Hence, this correction should not be relevant to the present $H_{0}$ tension problem.

\section{B. Corrections to the Kepler problem}

By the Kepler problem, one means the motion of a body under the central $\frac{1}{r}$ potential. In reality, an additional inverse cubic term should be added to account for the general relativity corrections. Here, only the modifications to the $\frac{1}{r}$ term arising from noncommutativity will be considered (in first $\ell^{2}$ order). First, one considers the corrections to the classical Hamiltonian

$$
H=\frac{p^{2}}{2 m}+\frac{G}{r}
$$

Using the representation (11) and taking expectation values in a basis $e^{i r \cdot \frac{\bar{p}}{\bar{s}}}$ ( $r$ here is a c-number, not an operator),

$$
\begin{aligned}
\sum_{i}\left(e^{i r \cdot \frac{\bar{p}}{\tilde{s}}}, x^{i} x^{i} e^{i r \cdot \frac{\bar{p}}{\bar{s}}}\right)= & \sum_{i}\left(\left(r_{i}+\epsilon \ell^{2} p^{i} \frac{r \cdot \bar{p}}{\bar{\Im}^{2}}\right) e^{i r \cdot \frac{\bar{p}}{\bar{\xi}}}\right. \\
& \left.\left(r_{i}+\epsilon \ell^{2} p^{i} \frac{r \cdot \bar{p}}{\bar{\Im}^{2}}\right) e^{i r \cdot \frac{\bar{p}}{\bar{s}}}\right) \\
= & |r|^{2}\left(1-2 \epsilon \ell^{2} \frac{(\hat{r} \cdot \bar{p})^{2}}{\bar{\Im}^{2}}\right)+O\left(\ell^{4}\right),
\end{aligned}
$$

one sees that the $O\left(\ell^{2}\right)$ corrections to the classical Hamiltonian (53) amount to the replacement

$$
\frac{G}{r} \rightarrow \frac{G}{r}\left(1+\epsilon \ell^{2}\left(\hat{r} \cdot \frac{\bar{p}}{\bar{s}}\right)^{2}+O\left(\ell^{4}\right)\right),
$$


where $\hat{r}=\frac{r}{|r|}$. One obtains a positive or negative correction (depending on $\epsilon$ ) of the coupling constant. For classical bound quasicircular orbits, $\left(\hat{r} \cdot \frac{\bar{p}}{\bar{J}}\right)$ is very small; therefore, any detectable corrections to the classical motion could only be expected for flyby orbits.

With the estimate

$$
\int_{\text {collision }}(\hat{r} \cdot \bar{p})^{2}=\int_{0}^{\pi}|\bar{p}|^{2} \cos ^{2} \theta d \theta=|\bar{p}|^{2} \frac{\pi}{2},
$$

the approximate correction to the coupling constant would be

$$
G \rightarrow G\left(1+\epsilon \ell^{2}|\bar{p}|^{2} \frac{\pi}{2}\right)
$$

which for a macroscopic speed $15 \mathrm{~km} / \mathrm{sec}$, the proton mass and $\ell=10^{-19} \mathrm{~cm}$ leads to

$$
\ell^{2}|\bar{p}|^{2} \frac{\pi}{2}=8.88 \times 10^{-20},
$$

much too small to be observable.

Next, one computes the modifications to the quantum Coulomb spectrum arising from Eq. (54). Because

$$
\frac{1}{2}(\hat{r} \cdot \bar{p}+\bar{p} \cdot \hat{r})=p_{r}=\frac{1}{i} \frac{1}{r} \frac{\partial}{\partial r} r,
$$

the radial equation becomes (in leading $\ell^{2}$ order)

$$
\begin{aligned}
& \left\{\left(1-\frac{2 \epsilon \ell^{2} m e^{2}}{r} Z\right) \frac{d^{2}}{d r^{2}}-\frac{L(L+1)}{r^{2}}+\frac{2 m e^{2}}{r} Z+2 m e E\right\} \\
& \quad \times r \psi(r)=0 .
\end{aligned}
$$

One now considers the eigenstates of the unperturbed equation and treats the term $\Delta=\frac{2 \epsilon \ell^{2} m e^{2}}{r} Z \frac{d^{2}}{d r^{2}} r$ acting on $\psi(r)$ as a perturbation. Because of the $\frac{1}{r}$ factor in $\Delta$, one expects the largest effects to occur for $s$ states. One obtains for the first and second $s$ states

$$
\begin{aligned}
& \left\langle\psi_{1 s}, \Delta \psi_{1 s}\right\rangle=-3 \epsilon \ell^{2} Z^{4} m^{3} e^{8}, \\
& \left\langle\psi_{2 s}, \Delta \psi_{2 s}\right\rangle=-\frac{7}{16} \epsilon \ell^{2} Z^{4} m^{3} e^{8},
\end{aligned}
$$

and denoting by $H_{0}$ the unperturbed Hamiltonian, with $\left\langle\psi_{n s}, H_{0} \psi_{n s}\right\rangle=-\frac{Z^{2} m e^{4}}{2 n^{2}}$,

$$
\begin{aligned}
& \frac{\left\langle\psi_{1 s}, \Delta \psi_{1 s}\right\rangle}{\left\langle\psi_{1 s}, H_{0} \psi_{1 s}\right\rangle}=6 \epsilon \ell^{2} Z^{2} m^{2} e^{4}, \\
& \frac{\left\langle\psi_{2 s}, \Delta \psi_{2 s}\right\rangle}{\left\langle\psi_{2 s}, H_{0} \psi_{2 s}\right\rangle}=\frac{7}{2} \epsilon \ell^{2} Z^{2} m^{2} e^{4} .
\end{aligned}
$$

Of more significance is perhaps the mixing matrix element,

$$
\left\langle\psi_{2 s}, \Delta \psi_{1 s}\right\rangle=-\frac{44}{27} \epsilon \ell^{2} Z^{4} m^{3} e^{8} .
$$

If $m=m_{e}$, the electron mass, and $\ell=10^{-19} \mathrm{~cm}$, then

$$
m_{e}^{2} \ell^{2} e^{4}=0.357 \times 10^{-21} .
$$

However, for muon atoms and large $Z$, this value is multiplied by a factor $\approx(200 \times Z)^{2}$.

For other noncommutative corrections to the Coulomb problem, refer to Ref. [26], where, in particular, angular momentum effects were taken into consideration.

\section{Phase-space volume effects}

The phase-space contraction for $\epsilon=+1$ and the phasespace expansion for $\epsilon=-1$ have already been described in Refs. [24,26]. Here, I simply rederive this result in the context of the general representation (11) and update the experimental perspectives.

Consider a particular space coordinate $x^{i} \stackrel{\circ}{=} x, \bar{p}^{i} \stackrel{\circ}{=} p$. Then,

$$
x=i\left(\epsilon \ell^{2} p \frac{\partial}{\partial \bar{\Im}}+\bar{\Im} \frac{\partial}{\partial p}\right) .
$$

The eigenstates of this operator are

$$
|x\rangle=\exp \left(-i \frac{x}{\ell} \tanh ^{-1}\left(\frac{\ell p}{\overline{\mathfrak{s}}}\right)\right)
$$

for $\epsilon=+1$ and

$$
|x\rangle=\exp \left(-i \frac{x}{\ell} \tan ^{-1}\left(\frac{\ell p}{\bar{\Im}}\right)\right)
$$

for $\epsilon=-1$.

To obtain the wave function of a momentum wave function on the $|x\rangle$ basis $(\epsilon=+1)$, one projects by integration on the $p, \bar{\Im}$ variables:

$$
\langle x \mid k\rangle=\int J(p, \bar{\Im}) d p d \bar{\Im} e^{i \frac{x}{t} \tanh ^{-1}\left(\frac{e p}{\Im}\right)} \delta(p-k),
$$

with $J(p, \bar{\Im})$ being an integration density. To proceed, it is convenient to change variables to

$$
\begin{aligned}
& x=i \ell \frac{\partial}{\partial \mu}, \\
& p=\frac{R}{\ell} \sinh \mu, \\
& \bar{\Im}=R \cosh \mu
\end{aligned}
$$

and convert Eq. (58) into 


$$
\begin{aligned}
\langle x \mid k\rangle & =\int d R d \mu e^{i \frac{x}{t} \mu} \delta\left(\mu-\sinh ^{-1}\left(\frac{\ell k}{R}\right)\right) \delta(R-1) \\
& =e^{i \frac{i x}{\ell} \sinh ^{-1}(\ell k)} .
\end{aligned}
$$

The choice $R=1$ corresponds in Eq. (59) to the choice of a particular representation of the pseudo-Euclidean algebra in two dimensions. It corresponds to the choice of a density $J(p, \bar{\Im})$ :

$$
J(p, \bar{\Im})=\delta\left(\bar{\Im}-\sqrt{1+\ell^{2} p^{2}}\right) .
$$

For $\epsilon=-1$, a similar calculation leads to

$$
\langle x \mid k\rangle=e^{i \frac{x}{\ell} \sin ^{-1}(\ell k)} .
$$

The density of states is obtained from

$$
\begin{aligned}
\frac{x+L}{\ell} \sinh ^{-1}\left(\ell k_{n}\right) & =\frac{x}{\ell} \sinh ^{-1}(\ell k)+2 \pi n, \\
\frac{x+L}{\ell} \sin ^{-1}\left(\ell k_{n}\right) & =\frac{x}{\ell} \sin ^{-1}(\ell k)+2 \pi n,
\end{aligned}
$$

leading to

$$
\begin{array}{ll}
d n=\frac{L}{2 \pi} \frac{d k}{\sqrt{1+\ell^{2} p^{2}}} & \text { for } \epsilon=+1, \\
d n=\frac{L}{2 \pi} \frac{d k}{\sqrt{1-\ell^{2} p^{2}}} \quad \text { for } \epsilon=-1 .
\end{array}
$$

For three dimensions [24],

$$
\begin{array}{ll}
d n=\frac{V}{2 \pi^{2}} \frac{1}{\ell^{2}} \frac{\left(\sinh ^{-1}(\ell|p|)\right)^{2} d k}{\sqrt{1+\ell^{2}|p|^{2}}} \text { for } \epsilon=+1, \\
d n=\frac{V}{2 \pi^{2}} \frac{1}{\ell^{2}} \frac{\left(\sin ^{-1}(\ell|p|)\right)^{2} d k}{\sqrt{1-\ell^{2}|p|^{2}}} \quad \text { for } \epsilon=-1 .
\end{array}
$$

As discussed in Refs. [24,26], the contraction or expansion of the phase space has an impact on the cross sections of elementary processes. For example, for the cross section of the reaction

$$
\gamma+p \rightarrow \pi+N
$$

of high-energy proton cosmic rays, the contraction of phase space in the $\epsilon=+1$ case would allow cosmic-ray protons of higher energies and from further distances to reach the Earth. From the calculations performed in Ref. [26], one knows that the phase-space suppression factor for the photon pion production is a function of $\alpha=\omega_{\gamma}^{\prime 2} \ell^{2}$, with $\omega_{\gamma}^{\prime}$ being the photon energy in the proton rest frame, the suppression being only appreciable if $\alpha \succeq 1$. In this case, $\omega_{\gamma}^{\prime}=1.49 \times 10^{-12} p_{P}^{0}, p_{P}^{0}$ being the proton energy. Therefore, for this reaction the effect would be very small for $\ell=O\left(10^{-19} \mathrm{~cm}\right)$. In any case, even for larger values of $\ell$, the GZK cutoff would not be much changed, the main difference being a bigger size for the GZK sphere, meaning that more cosmic-ray protons from further distances would be able to reach the Earth.

A better place to look for the effects of this phase-space suppression might be an increase $(\epsilon=+1)$ or decrease $(\epsilon=-1)$ in particle multiplicity in high-energy reactions [24]. This effect would be important when $\ell k \sim O(1), k$ being the typical reaction momentum. For $\ell=10^{-19} \mathrm{~cm}$, this would occur for $k \approx 100-200 \mathrm{TeV}$ (in the range of the future FCC).

\section{Diffraction, interference, and uncertainty relations}

Massless or massive wave equations in the noncommutative context are solutions of [22]

$$
\left[p^{\mu},\left[p_{\mu}, \psi\right]\right]=0
$$

or

$$
\left[p^{\mu},\left[p_{\mu}, \psi\right]\right]-m^{2} \psi=0,
$$

where $\psi$ may be either a scalar or a tensor element of the enveloping algebra $U\left(\Re_{\ell, \infty}\right)$ of the algebra $\Re_{\ell, \infty}=$ $\left\{x^{\mu}, M^{\mu \nu}, \bar{p}^{\mu}, \bar{\Im}\right\}$. They have a general solution

$$
\psi_{k}(x)=\exp \left(i k \cdot \frac{1}{2}\left\{x, \bar{\Im}^{-1}\right\}_{+}\right),
$$

from which quantum fields may be constructed [22] with $k^{2}=0$ or $m^{2}$. Notice that in Eq. (66), the $x^{\mu}$ 's are simply algebra elements, not the coordinates of the wave. Physical results are obtained from the application of a state to the algebra.

From the commutator $\left[\bar{p}^{\mu}, x^{\nu}\right]=i \eta^{\mu \nu} \bar{\Im}$, it also follows that the wave equations also have factorized solutions:

$$
\psi_{k}(x)=\prod_{\mu=0}^{3} \psi_{k^{\mu}}\left(x^{\mu}\right),
$$

with

$$
\psi_{k^{\mu}}\left(x^{\mu}\right)=e^{i \frac{1}{2} k^{\mu}\left\{x_{\mu}, \bar{\jmath}^{-1}\right\}_{+}} \quad(\text { fixed } \mu) .
$$

The factorized solutions may be used to study the diffraction problem. A geometry is chosen with one or two long slits along the $x^{2}$ coordinate and an incident wave along the third coordinate, $\vec{k}=k \vec{e}_{3}$. The wave in the slit(s) will be a superposition of localized states on the first space coordinate $x^{1}$, namely (for a single slit) of width $2 \Delta$ (in the $\bar{p}^{1}, \bar{\Im}$ representation): 


$$
\left|\chi_{1}\right\rangle_{+}=\int_{-\Delta}^{\Delta} d x^{1} e^{-i \frac{x^{1}}{e} \tanh ^{-1}\left(\frac{\ell p^{1}}{\grave{s}}\right)}
$$

for $\epsilon=+1$ and

$$
\left|\chi_{1}\right\rangle_{-}=\int_{-\Delta}^{\Delta} d x^{1} e^{-i \frac{x^{1}}{\ell} \tan ^{-1}\left(\frac{\ell p^{1}}{\Im}\right)}
$$

for $\epsilon=-1$. Therefore, after passing the slit, the wave is

$$
|\Psi\rangle_{k}=\psi_{k^{0}}\left(x^{0}\right) \int d \xi\left\langle\psi_{\xi}\left(x^{1}\right) \mid \chi\right\rangle \psi_{\xi}\left(x^{1}\right) \psi \sqrt{k^{2}-\xi^{2}}\left(x^{3}\right) .
$$

The projections $\left\langle\psi_{\xi}\left(x^{1}\right) \mid \chi\right\rangle$ of the slit state on the wave equation solution $\psi_{\xi}\left(x^{1}\right)$ will be computed in order $\ell^{2}$. In addition, because of the factorized nature of the solutions, one may use for the operators $x^{1}, p^{1}$, and $\bar{\Im}$ a subalgebra representation instead of the general representation (11), namely

$$
\begin{aligned}
x^{1} & =x, \\
p^{1} & =\frac{1}{\ell} \sinh \left(\frac{\ell}{i} \frac{d}{d x}\right), \quad \epsilon=+1, \\
\bar{\Im} & =\cosh \left(\frac{\ell}{i} \frac{d}{d x}\right), \\
x^{1} & =x, \\
p^{1} & =\frac{1}{\ell} \sin \left(\frac{\ell}{i} \frac{d}{d x}\right), \quad \epsilon=-1 . \\
\bar{\Im} & =\cos \left(\frac{\ell}{i} \frac{d}{d x}\right),
\end{aligned}
$$

Then, in $O\left(\ell^{2}\right)$,

$$
\bar{\Im}^{-1}=1-\epsilon \frac{1}{2}\left(\frac{\ell}{i}\right)^{2} \frac{d^{2}}{d x^{2}}+\frac{5}{4}\left(\frac{\ell}{i}\right)^{4} \frac{d^{4}}{d x^{4}}-\cdots,
$$

and in the representations (72) and (73), the generalized localized states are simply $\left|\chi_{1}\right\rangle=\delta\left(x^{1}-\eta\right)$. The projection $\left\langle\psi_{\xi}\left(x^{1}\right) \mid \chi\right\rangle$ becomes

$$
\begin{aligned}
\left\langle\psi_{\xi}\left(x^{1}\right) \mid \chi\right\rangle & =\frac{1}{2 \pi} \int_{-\Delta}^{\Delta} e^{i \frac{1}{2} \xi\left\{x^{1}, \overline{\mathcal{J}}^{-1}\right\}} \delta\left(x^{1}-\eta\right) d \eta \\
& =\frac{1}{\pi} e^{-\epsilon_{4}^{1} \xi^{2} \ell^{2}} \frac{\sin \left(\Delta \xi\left(1-\epsilon \frac{1}{6} \xi^{2} \ell^{2}\right)\right)}{\xi-\epsilon \frac{1}{6} \xi^{3} \ell^{2}}+O\left(\ell^{4}\right) .
\end{aligned}
$$

Therefore, the intensity of the diffracted wave at angle $\theta=\sin ^{-1} \frac{\xi}{k}$ is proportional to

$$
\frac{\sin ^{2}\left(\Delta \xi\left(1-\epsilon \frac{1}{6} \xi^{2} \ell^{2}\right)\right)}{\left(\Delta \xi\left(1-\epsilon \frac{1}{6} \xi^{2} \ell^{2}\right)\right)^{2}} .
$$

For two slits of width $2 \Delta$ at a distance $2 \Sigma$, the slit states would be

$$
\int_{-\Sigma-\Delta}^{-\Sigma+\Delta}+\int_{\Sigma-\Delta}^{\Sigma+\Delta} d x^{1}\left|\chi_{1}\right\rangle
$$

with normalized diffracted intensity

$$
\frac{\sin ^{2}\left(\Delta \xi\left(1-\epsilon \frac{1}{6} \xi^{2} \ell^{2}\right)\right) \sin ^{2}\left(\Sigma \xi\left(1-\epsilon \frac{1}{6} \xi^{2} \ell^{2}\right)\right)}{\Delta^{2} \Sigma^{2}\left(\xi\left(1-\epsilon \frac{1}{6} \xi^{2} \ell^{2}\right)\right)^{4}} .
$$

One sees that the effects of noncommutativity become important for $\xi \ell \sim O(1)$. For $\ell \sim 10^{-19} \mathrm{~cm}$, this would be $\xi \gtrsim 100 \mathrm{TeV}$.

On the other hand, the noncommutativity of the spacetime coordinates implies uncertainty relations on the simultaneous measurement of two space coordinates, or of one space and one time coordinate. From

$$
\left[x^{\mu}, x^{\nu}\right]=-i \epsilon \ell^{2} M^{\mu \nu},
$$

one obtains for $\Delta x^{\mu}=\left(\left\langle\psi\left|\left(x^{\mu}\right)^{2}\right| \psi\right\rangle-\left\langle\psi\left|x^{\mu}\right| \psi\right\rangle^{2}\right)^{\frac{1}{2}}$

$$
\Delta x^{\mu} \Delta x^{\nu} \geq \frac{1}{2} \ell^{2}\left\langle\psi\left|M^{\mu \nu}\right| \psi\right\rangle .
$$

In particular, one notices that there is no space-space uncertainty if $|\psi\rangle$ is spinless, but time-space uncertainty leads to observable effects.

\section{REMARKS AND CONCLUSIONS}

(1) Approaching the question of noncommutative spacetime from the point of view of deformation theory and the principle of stability of physical theories, the first important observation is the independence of the length scales of noncommutativity of the coordinates $(\ell)$ and of the momenta $\left(\phi^{-1}\right)$. The scale of $\phi^{-1}$ being associated with the noncommutativity of translations is naturally associated with gravity and the Planck length. However, the scale of $\ell$ might be larger, and it makes sense to launch an experimental effort to find upper bounds or even the value of this length scale. At the present time, in addition to a precise analysis of phenomena of cosmological origin and a refinement of the neutrino speed measurements, another possibility lies in phase-space modification effects on highenergy colliders.

(2) The estimates, performed here based on GRB data, point to values of $\ell$ in the range $10^{-19}-10^{-21} \mathrm{~cm}$ [or $\left.\tau \in\left(0.3 \times 10^{-29}-0.3 \times 10^{31} \mathrm{sec}\right)\right]$ favoring the higher part of this range. However, these estimates can only 
be taken as indicative or as establishing upper bounds because of the large uncertainties on the calculation of the spectral lags, on the statistics of the GRB pulses, and on the nature of the intrinsic spectral lags.

(3) The deformed $\Re_{\ell, \infty}=\left\{x^{\mu}, M^{\mu \nu}, \bar{p}^{\mu}, \bar{\Im}\right\}$ algebra also has some consequences concerning the structure of the fundamental interactions, in particular those that are associated with connection-valued fields. In particular, the additional dimension in the differential algebra may imply the existence of new interactions and states, as well as a new extended structure for the Dirac equation. These questions, not dealt with here, because they have a less direct experimental verification, are described elsewhere [22,24].

(4) In the context of deformation theory, the transition from classical to quantum mechanics appears as the stabilization of the unstable Poisson algebra to the stable Moyal algebra. At the level of general nonlinear functions of position and momentum, the corresponding Hilbert space algebra of operators is also stable, but the Heisenberg algebra itself,

$$
[p, x]=-i \mathbf{1} \quad \hbar=1,
$$

is not, because the $c$-number $\mathbf{1}$ commutes with both $p$ and $x$. Stabilization would then suggest a deformation to

$$
[x, \mathbf{1}]=i \epsilon \ell^{2} p, \quad[p, \mathbf{1}]=-i \epsilon^{\prime} \phi^{2} x,
$$

and generalizing to $x^{\mu}$ and $p^{\mu}$ together with compatibility with the Lorentz group would lead to the tangent space deformed algebra $\Re_{\ell, \infty}=$ $\left\{x^{\mu}, M^{\mu \nu}, \bar{p}^{\mu}, \bar{\Im}\right\}$. In conclusion, in the framework of stable theories, this algebra is already implicit in the transition to quantum mechanics.

(5) All calculations in the previous sections were carried out for the algebra of the (noncommutative) tangent space limit $\phi^{-1} \rightarrow \infty$. When the full deformed algebra in Eq. (3) is used, the noncommutativity of momenta in

$$
\left[p^{\mu}, p^{\nu}\right]=-i \epsilon^{\prime} \phi^{2} M^{\mu \nu}
$$

corresponds to the noncommutativity of spacetime translations. A similar noncommutativity is what occurs in a gravitational field. In this sense, gravitation might also be considered an emergent property arising from deformation theory and the principle of stability of physical theories. Considering $\phi$ rather than the metric as defining gravitational field, gravitation would be formulated as a $S O(3,3)$ gauge theory [23]. An interesting consequence is that the gravitational field might be a function of the Casimir invariants of $S O(3,3)$, and not only of the energy-momentum tensor.

\section{APPENDIX: THE GENERAL DEFORMATIONS OF THE POINCARÉ-HEISENBERG ALGEBRA}

The Poincaré-Heisenberg algebra

$$
\begin{aligned}
{\left[M^{\mu \nu}, M^{\rho \sigma}\right] } & =i\left(M^{\mu \sigma} \eta^{\nu \rho}+M^{\nu \rho} \eta^{\mu \sigma}-M^{\nu \sigma} \eta^{\mu \rho}-M^{\mu \rho} \eta^{\nu \sigma}\right), \\
{\left[M^{\mu \nu}, p^{\lambda}\right] } & =i\left(p^{\mu} \eta^{\nu \lambda}-p^{\nu} \eta^{\mu \lambda}\right), \\
{\left[M^{\mu \nu}, x^{\lambda}\right] } & =i\left(x^{\mu} \eta^{\nu \lambda}-x^{\nu} \eta^{\mu \lambda}\right), \\
{\left[p^{\mu}, x^{\nu}\right] } & =i \eta^{\mu \nu} \\
{\left[x^{\mu}, x^{\nu}\right] } & =0 \\
{\left[p^{\mu}, p^{\nu}\right] } & =0
\end{aligned}
$$

is not stable (rigid). Its 2-cohomology group has three nontrivial generators, which lead to the following modified commutators $^{7}[18]$ :

$$
\begin{aligned}
{\left[p^{\mu}, x^{\nu}\right] } & =i \eta^{\mu \nu} \Im+i \beta_{3} M^{\mu \nu}, \\
{\left[x^{\mu}, x^{\nu}\right] } & =i \beta_{2} M^{\mu \nu}, \\
{\left[p^{\mu}, p^{\nu}\right] } & =i \beta_{1} M^{\mu \nu} \\
{\left[x^{\mu}, \Im\right] } & =-i \beta_{2} p^{\mu}+i \beta_{3} x^{\mu}, \\
{\left[p^{\mu}, \Im\right] } & =i \beta_{1} x^{\mu}-i \beta_{3} p^{\mu}, \\
{\left[M^{\mu \nu}, \Im\right] } & =0 .
\end{aligned}
$$

There is an instability cone at $\beta_{3}^{2}=\beta_{1} \beta_{2}$, but for generic $\beta_{1}$, $\beta_{2}, \beta_{3}$ all these algebras are rigid and are isomorphic to either $S O(1,5)$ or $S O(2,4)$ or $S O(3,3)$ (depending on the signs of $\beta_{1}$ and $\beta_{2}$ ). For all these classes, there is a representative with $\beta_{3}=0$, which is exactly the deformation (3) obtained in Ref. [16]. The $\beta_{3}=0$ situation may always be obtained by a linear change of coordinates in the algebra. The converse situation $\beta_{1}=\beta_{2}=0$ and $\beta_{3} \neq 0$ also mentioned in Ref. [16] leads to $\left[x^{\mu}, x^{\nu}\right]=\left[p^{\mu}, p^{\nu}\right]=0$, which does not seem to be physically relevant, because at least the second commutator is expected to be different from zero in the presence of gravity.

Here and elsewhere, I will be interpreting $x^{\mu}$ and $p^{\nu}$ as the physical coordinates and momenta. In this sense, I do not agree with the criticism in Ref. [18] about this choice, because not all observables have to be extensive, only those that correspond to symmetry transformations, in this case $M_{\mu \nu}$ and $p_{\mu}$.

\footnotetext{
${ }^{7}$ In the notation of Ref. [18], $\beta_{i}=q \alpha_{i}$ and $\mathfrak{\Im}=q M$.
} 
[1] H. S. Snyder, Quantized spacetime, Phys. Rev. 71, 38 (1947); The electromagnetic field in quantized spacetime, Phys. Rev. 72, 68 (1947).

[2] C. N. Yang, On quantized spacetime, Phys. Rev. 72, 874 (1947).

[3] M. R. Douglas and N. A. Nekrasov, Noncommutative field theory, Rev. Mod. Phys. 73, 977 (2001).

[4] L. Alvarez-Gaumé and M. A. Vazquez-Mozo, General properties of noncommutative field theory, Nucl. Phys. B668, 293 (2003).

[5] I. Hinchliffe, N. Kersting, and Y. L. Ma, Review of the phenomenology of noncommutative geometry, Int. J. Mod. Phys. A 19, 179 (2004).

[6] S. Hossenfelder, Minimal length scale scenarios for quantum gravity, Living Rev. Relativity 16, 2 (2013).

[7] D. V. Ahluwalia, Quantum measurement, gravitation, and locality, Phys. Lett. B 339, 301 (1994).

[8] S. Doplicher, K. Fredenhagen, and J.E. Roberts, The quantum structure of spacetime at the Planck scale and quantum fields, Commun. Math. Phys. 172, 187 (1995).

[9] G. Amelino-Camelia, Quantum-spacetime phenomenology, Living Rev. Relativity 16, 5 (2013).

[10] R. Vilela Mendes, The stability of physical theories principle, in Beyond Peaceful Coexistence: The Emergence of Space, Time and Quantum, edited by I. Licata (Imperial College Press, London, 2016), pp. 153-200.

[11] A. Andronov and L. Pontryagin, Systèmes grossiers, Dokl. Akad. Nauk. USSR 14, 247 (1937).

[12] S. Smale, Differentiable dynamical systems, Bull. Am. Math. Soc. 73, 747 (1967).

[13] M. Flato, Deformation view of physical theories, Czech J. Phys. B 32, 472 (1982).

[14] L. D. Faddeev, On the relationship between mathematics and physics, Asia-Pac. Phys. News 3, 21 (1988); Frontiers in Physics, High Technology and Mathematics, edited by H. A. Cerdeira and S. O. Lundqvist (World Scientific, Singapore, 1989), pp. 238-246.

[15] F. Bayen, M. Flato, C. Fronsdal, C. Lichnerowicz, and D. Sternheimer, Deformation theory and quantization, Ann. Phys. (N.Y.) 111, 61 (1978).

[16] R. Vilela Mendes, Deformations, stable theories and fundamental constants, J. Phys. A 27, 8091 (1994).

[17] R. Vilela Mendes, Quantum mechanics and noncommutative spacetime, Phys. Lett. A 210, 232 (1996).

[18] C. Chryssomalakos and E. Okon, Generalized quantum relativistic kinematics: A stability point of view, Int. J. Mod. Phys. D 13, 2003 (2004).

[19] D. V. Ahluwalia-Khalilova, Minimal spatio-temporal extent of events, neutrinos, and the cosmological constant problem, Int. J. Mod. Phys. D 14, 2151 (2005).

[20] D. V. Ahluwalia-Khalilova, A freely falling frame at the interface of gravitational and quantum realms, Classical Quantum Gravity 22, 1433 (2005).

[21] K. Morita, Discrete symmetries in Lorentz-invariant noncommutative QED, Prog. Theor. Phys. 110, 1003 (2003).

[22] R. Vilela Mendes, Geometry, stochastic calculus and quantum theories in a noncommutative spacetime, J. Math. Phys. (N.Y.) 41, 156 (2000).

[23] R. Vilela Mendes, The geometry of noncommutative spacetime, Int. J. Theor. Phys. 56, 259 (2017).
[24] R. Vilela Mendes, A laboratory scale fundamental time, Eur. Phys. J. C 72, 2239 (2012).

[25] R. Vilela Mendes, An extended Dirac equation in noncommutative spacetime, Mod. Phys. Lett. A 31, 1650089 (2016).

[26] R. Vilela Mendes, Some consequences of a noncommutative spacetime structure, Eur. Phys. J. C 42, 445 (2005).

[27] R. Vilela Mendes, The deformation-stability fundamental length and deviations from $c$, Phys. Lett. A 376, 1823 (2012).

[28] T. Adam et al., Measurement of the neutrino velocity with the OPERA detector in the CNGS beam, J. High Energy Phys. 10 (2012) 093.

[29] E. Carlen and R. Vilela Mendes, Noncommutative spacetime and the uncertainty principle, Phys. Lett. A 290, 109 (2001).

[30] P. Adamson et al., Measurement of neutrino velocity with the MINOS detectors and NuMI neutrino beam, Phys. Rev. D 76, 072005 (2007).

[31] K. Hirata et al., Observation of a Neutrino Burst from the Supernova SN 1987A, Phys. Rev. Lett. 58, 1490 (1987).

[32] R. Bionta et al., Observation of a Neutrino Burst in Coincidence with Supernova SN 1987a in the Large Magellanic Cloud, Phys. Rev. Lett. 58, 1494 (1987).

[33] M. J. Longo, Tests of relativity from SN 1987A, Phys. Rev. D 36, 3276 (1987).

[34] G. Amelino-Camelia, J. Ellis, N.E. Mavromatos, D. V. Nanopoulos, and S. Sarkar, Tests of quantum gravity from observations of gamma-ray bursts, Nature (London) 393, 763 (1998).

[35] H. Xu and B. Q. Ma, Regularity of high energy photon events from gamma ray bursts, J. Cosmol. Astropart. Phys. 01 (2018) 050.

[36] J. Ellis, R. Konoplich, N. E. Mavromatos, L. Nguyen, A. S. Sakharov, and E. K. Sarkisyan-Grinbaum, Robust constraint on Lorentz violation using Fermi-LAT gamma ray burst data, Phys. Rev. D 99, 083009 (2019).

[37] J.P. Norris, G. F. Marani, and J. T. Bonnell, Connection between energy-dependent lags and peak luminosity in gamma-ray bursts, Astrophys. J. 534, 248 (2000).

[38] J. P. Norris, Implications of the lag-luminosity relationship for unified gamma-ray burst paradigms, Astrophys. J. 579, 386 (2002).

[39] T. N. Ukwatta, M. Stamatikos, K. S. Dhuga, T. Sakamoto, S. D. Barthelmy, A. Eskandarian, N. Gehrels, L.C. Maximon, J. P. Norris, and W. C. Parke, Spectral lags and the lag-luminosity relation: An investigation with Swift Bat gamma-ray bursts, Astrophys. J. 711, 1073 (2010).

[40] T. N. Ukwatta et al., The lag-luminosity relation in the GRB source-frame: An investigation with Swift Bat bursts, Mon. Not. R. Astron. Soc. 419, 614 (2012).

[41] L. Shao et al., A new measurement of the spectral lag of gamma-ray bursts and its implications for spectral evolution behaviors, Astrophys. J. 844, 126 (2017).

[42] S. Zhang and B.-Q. Ma, Lorentz violation from gamma-ray bursts, Astropart. Phys. 61, 108 (2015).

[43] M. G. Bernardini et al., Comparing the spectral lag of short and long gamma-ray bursts and its relation with luminosity, Mon. Not. R. Astron. Soc. 446, 1129 (2015). 
[44] J.-J. Wei and X.-F. Wu, A further test of Lorentz violation from the rest-frame spectral lags of gamma-ray bursts, Astrophys. J. 851, 127 (2017).

[45] M. G. Bernardini et al., Limits on quantum gravity effects from Swift short gamma-ray bursts, Astron. Astrophys. 607, A121 (2017).

[46] IceCube Collaboration, Neutrino emission from the direction of the blazar TXS 0506+056 prior to the IceCube170922A alert, Science 361, 147 (2018).

[47] IceCube Collaboration, Multimessenger observations of a flaring blazar coincident with high-energy neutrino IceCube-170922A, Science 361, eaat1378 (2018).

[48] R. Abbasi et al., An absence of neutrinos associated with cosmic-ray acceleration in $\gamma$-ray bursts, Nature (London) 484, 351 (2012).

[49] S. Adrián-Martinez et al., Search for muon neutrinos from gamma-ray bursts with the ANTARES neutrino telescope using 2008 to 2011 data, Astron. Astrophys. 559, A9 (2013).

[50] M. G. Aartsen et al., Search for prompt neutrino emission from gamma-ray bursts with IceCube, Astrophys. J. 805, L5 (2015).

[51] J.-J. Wei, B.-B. Zhang, L. Shao, X.-F. Wu, and P. Mészáros, A new test of Lorentz invariance violation: The spectral lag transition of GRB160625B, Astrophys. J. Lett. 834, L13 (2017).

[52] J.-J. Wei, X.-F. Wu, B.-B. Zhang, L. Shao, P. Mészáros, and V.A. Kostelecky, Constraining anisotropic Lorentz violation via the spectral lag transition of GRB160625B, Astrophys. J. 842, 115 (2017).

[53] S. Ganguly and S. Desai, Statistical significacnce of spectral lag transition in GRB160625B, Astropart. Phys. 94, 17 (2017). 